\title{
ANÁLISE DE RISCO EM QUATRO SISTEMAS DE ROTACÃO DE CULTURAS PARA TRIGO, NUM PERÍODO DE DEZ ANOS, EM PASSO FUNDO, RS ${ }^{1}$
}

\author{
HENRIQUE PEREIRA DOS SANTOS ${ }^{2}$, IVO AMBROSI ${ }^{3}$ e JULIO CESAR BARRENECHE LHAMBY ${ }^{4}$
}

RESUMO - Num período de dez anos (1980 a 1989), foi conduzido, na Embrapa-Centro Nacional de Pesquisa de Trigo (CNPT), em Passo Fundo, RS, experimento reunindo quatro sistemas de rotação de culturas para trigo (Triticum aestivum L.): sistema I (trigo/soja); sistema II (trigo/soja, colza/soja, cevada/soja e tremoço ou serradela/milho); sistema III (trigo/soja, trevo vesiculoso/trevo vesiculoso, trevo vesiculoso/milho, de 1980 a 1983, e trigo/soja, aveia branca/soja e ervilhaca/milho, de 1984 a 1989); e sistema IV (trigo/soja, colza/soja, linho/soja e tremoço ou serradela/milho). Utilizou-se o delineamento de blocos ao acaso, com quatro repetições e parcelas com área útil de $120 \mathrm{~m}^{2}$. No presente trabalho, mostra-se a análise de risco naquele período. Foram aplicados dois tipos de análise na receita líquida dos sistemas: análise da média variância e análise de risco (distribuição de probabilidade acumulada e dominância estocástica). Pela análise da média variância da receita líquida, os sistemas II e IV foram superiores aos sistemas I e III. Pelo método da dominância estocástica, o sistema II apresentouse como a melhor alternativa de produção a ser oferecida aos agricultores, do ponto de vista de lucratividade e menor risco.

Termos para indexação: Triticum aestivum, análise da receita líquida, média variância, dominância estocástica.

\section{RISK ANALYSIS OF FOUR CROP ROTATION SYSTEMS FOR WHEAT, OVER A TEN-YEAR PERIOD, IN PASSO FUNDO, RS}

\begin{abstract}
During ten years (1980 to 1989), at the Embrapa-Centro Nacional de Pesquisa de Trigo (CNPT), in Passo Fundo, RS, Brazil, the effects of crop rotation systems on wheat (Triticum aestivum L.) were assessed. Four rotation systems for wheat were studied: system I (wheat/soybean); system II (wheat/soybean, rapeseed/soybean, barley/soybean, lupine or serradella/corn); system III (wheat/soybean, arrowleaf clover/arrowleaf clover, and arrowleaf clover/corn, from 1980 to 1983, and wheat/soybean, white oats/soybean, and common vetch/corn, from 1984 to 1989); and system IV (wheat/soybean, rapeseed/soybean, flax/soybean, and lupine or serradella/corn). A randomized complete block design, with four replications and plots with $120 \mathrm{~m}^{2}$, was used. Risk analysis over that period is presented in this paper. Two types of analysis were applied to the net return of the systems: mean-variance analysis and risk analysis (safety-first and stochastic dominance). By the mean-variance analysis, systems II and IV showed higher net returns as compared to systems I and III. The system II showed the highest profit and the lowest risk to be offered to the farmer, as compared to the remaining systems studied.
\end{abstract}

Index terms: Triticum aestivum, net return, mean-variance analysis, stochastic dominance.

\footnotetext{
${ }^{1}$ Aceito para publicação em 3 de agosto de 1998.

${ }^{2}$ Eng. Agr., Dr., Embrapa-Centro Nacional de Pesquisa de Trigo (CNPT), Caixa Postal 569, CEP 99001-970 Passo Fundo, RS. Bolsista do CNPq. E-mail: hpsantos@sede.embrapa.br

${ }^{3}$ Economista, M.Sc., Embrapa-CNPT.

${ }^{4}$ Eng. Agr., Dr., Embrapa-CNPT.
}

\section{INTRODUÇÃO}

A rotação de culturas, como prática corrente na produção, tem sido reconhecida do ponto de vista técnico como um dos meios indispensáveis ao bom desenvolvimento de uma agricultura estável (Reunião de Pesquisa de Soja da Região Sul, 1995). Em 
diversos estudos podem ser constatados os efeitos benéficos da rotação de culturas, tanto sobre as condições de solo quanto sobre a produção das culturas subseqüentes (Reis et al., 1983; Derpsch et al., 1991; Santos \& Reis, 1991; Santos \& Pereira, 1994). Essa prática determina inúmeras vantagens ao agricultor, com destaque para o aumento da produtividade.

A rotação de culturas, que resulta em diversificação e em diminuição de risco (Silva \& Dhein, 1994), pode ser afetada por variáveis naturais incontroláveis (quantidade e distribuição de precipitações pluviais e variações de temperatura) que interferem no desenvolvimento das espécies. Além disso, existe um risco econômico ou de mercado derivado de mudanças no preço dos produtos ou dos insumos e nas oportunidades de mercado (Ambrosi \& Zentner, 1991).

Deve ser levado em conta que o risco está presente em quase todas as atividades agrícolas e que o agricultor, de forma intuitiva, considera-o em suas tomadas de decisão (Moutinho et al., 1978). A incorporação da análise de risco à avaliação econômica é um procedimento que pode proporcionar, tanto aos economistas como aos pesquisadores, a melhor alternativa de produção a ser oferecida aos agricultores (Ambrosi \& Fontaneli, 1994).

De acordo com Cruz (1986), vários modelos de análise da receita líquida podem ser utilizados na incorparação de risco, em experimentação agrícola. Um dos que mais se adapta à experimentação agrícola é a análise da média variância (Feldstein, 1969), que considera apenas os dois primeiros momentos da distribuição dos retornos ou da renda e a análise de risco propriamente dita (distribuição de probabilidade acumulada sugere o critério da segurança em primeiro lugar e da dominância estocástica leva em conta toda a distribuição cumulativa de probabilidade dos retornos) (Hanoch \& Levy, 1970; Anderson, 1976).

No caso de experimentos de longa duração (rotação de culturas, sistema de produção de grãos ou sistema de produção misto) há relativamente poucos trabalhos no Brasil aplicando a análise da média variância ou a análise de risco. Ambrosi \& Fontaneli (1994), pesquisando sistemas de produção mistos (lavoura + pecuária), observaram, pela análise da dominância estocástica, que o cultivo de trigo/soja, aveia preta + ervilhaca pastejada/soja e aveia preta + ervilhaca pastejada/milho, foi a melhor alternativa de produção, do ponto de vista de rentabilidade e menor risco.

Santos et al. (1995b), trabalhando com sistemas de rotação de culturas para cevada, verificaram, também pela análise da dominância estocástica, que cevada/soja e ervilhaca/milho, de 1984 a 1989, e cevada/soja e aveia branca/soja, de 1990 a 1993, foram os sistemas mais lucrativos e de menores riscos. Santos et al. (1995a), pesquisando sistemas de rotação de culturas para trigo, observaram, pela análise da média variância e de risco (distribuição de probabilidade acumulada e dominância estocástica), que os sistemas trigo/soja e ervilhaca/milho, de 1984 a 1989, e trigo/soja e aveia branca/soja, de 1990 a 1993, foram os que proporcionaram a melhor alternativa a ser oferecida aos agricultores.

O presente trabalho teve por objetivo avaliar, sob o ponto de vista econômico, o risco que o agricultor poderá correr na adoção de quatro sistemas de rotação de culturas para trigo.

\section{MATERIAL E MÉTODOS}

Os dados usados neste trabalho foram obtidos em experimentos de sistemas de rotação de culturas para trigo desenvolvidos na Embrapa-Centro Nacional de Pesquisa de Trigo (CNPT), em Passo Fundo, RS ( $28^{\circ} 15^{\prime} \mathrm{S}, 52^{\circ} 24^{\prime} \mathrm{W}$ e $684 \mathrm{~m}$ de altitude), de 1980 a 1989, em Latossolo Vermelho-Escuro distrófico (Brasil, 1973).

As análises da média variância e de risco foram determinadas em quatro sistemas de rotação de culturas para trigo: sistema I (trigo/soja); sistema II (trigo/soja, colza/soja, cevada/soja e tremoço ou serradela/milho); sistema III (trigo/soja, trevo vesiculoso/trevo vesiculoso e trevo vesiculoso/milho, de 1980 a 1983, e trigo/soja, aveia branca/soja e ervilhaca/milho, de 1984 a 1989); e sistema IV (trigo/soja, colza/soja, linho/soja e tremoço ou serradela/milho) (Tabela 1), de acordo com o descrito por Santos et al. (1990). Em 1980, no sistema II a cevada foi substituída pelo trigo e o milho pela soja; no sistema IV, o linho e o tremoço não foram semeados. Em 1986, nos sistemas II e IV, o tremoço foi substituído por serradela. O sistema II difere do sistema IV por estar incluída a cevada, que poderia transmitir doenças, do sistema radicular e da parte aérea, ao trigo. Deve ser levado em conta que o sistema I completa o ciclo a cada ano, os sistemas II e IV a cada quatro anos, e o sistema III a cada três anos. 
A adubação de manutenção foi realizada de acordo com a recomendação para cada cultura e baseada nos resultados da análise de solo. As amostras de solo foram coletadas após as colheitas das culturas de inverno e de verão. Em 1981 e em 1987, antes da semeadura de inverno, foram aplicadas, respectivamente, 9,0 e 7,3 tha ${ }^{-1}$ de calcário com PRNT de $56 \%$ e de $70 \%$.

As culturas de inverno e o milho, de 1980 a 1983, foram estabelecidos em preparo convencional de solo, e a soja (1980 a 1989) e o milho, de 1984 a 1989, em plantio direto. A semeadura, o controle de plantas daninhas e os tratamentos fitossanitários foram realizados de acordo com a recomendação específica para cada cultura, e a colheita foi realizada com colhedeira especial de parcelas. Os rendimentos de cevada, de milho, de soja e de trigo foram corrigidos para umidade de $13 \%$, os de colza, para $9 \%$, e os de linho, para $10 \%$. O rendimento de grãos de cevada foi corrigido em função da classificação comercial (CEVACOR) (Ignaczak et al., 1980).

A análise econômica usada segue o trabalho de Zentner et al. (1990). Todos os preços foram convertidos para a moeda americana (US\$), cujo valor unitário na data do levantamento de preços, em novembro de 1990, era de CR\$ 128,60 . Deve ser levado em conta que os valores médios empregados nos preços e nas amostras representam valores constantes de novembro de 1990, eliminandose, desta forma, o problema da inflação. No caso de aveia branca, de ervilhaca, de serradela, de tremoço e de trevo, foi considerada como rendimento a contribuição ao solo de $90 \mathrm{~kg}$ de N/ha (Derpsch \& Calegari, 1992).

O delineamento experimental utilizado foi o de blocos ao acaso, com quatro repetições. A área útil da parcela foi de $20 \mathrm{~m}$ de comprimento por $10 \mathrm{~m}$ de largura $\left(200 \mathrm{~m}^{2}\right)$. Foi efetuada a análise de variância da receita líquida (média variância) da média conjunta dos anos (1980 a 1989). As médias foram comparadas entre si pela aplicação do teste de Duncan, a $5 \%$ de probabilidade. Entende-se por receita líquida a diferença entre a receita bruta (rendimento de grãos das espécies em estudo x preço de venda como produto comercial) e os custos totais (custos variáveis (custos dos insumos + custos das operações de campo) e custos fixos (depreciação de equipamentos, juros sobre o capital)).

Paralelamente, foi aplicado à receita líquida o programa para computador denominado Biorisco, ou Pacta, que é baseado no critério de simetria de Hanoch \& Levy (1970). Esse programa compara as alternativas, duas a duas, sob os pontos de vista de rentabilidade e de risco (distribuição de probabilidade acumulada, twentiles, e dominância estocástica, pairwise), conforme descrito por Cruz (1980).

\section{RESULTADOS E DISCUSSÃO}

Os rendimentos de grãos de cada espécie obtidos nos diferentes sistemas de rotação e nos diferentes anos são apresentados na Tabela 2.

TABELA 1. Sistemas de rotação de culturas para trigo com espécies de inverno e de verão. Embrapa-CNPT, Passo Fundo, RS, 1980 a 1989 1.

\begin{tabular}{|c|c|c|c|c|c|c|c|c|c|c|}
\hline \multirow{2}{*}{$\begin{array}{l}\text { Sistema } \\
\text { de rotação }\end{array}$} & \multicolumn{10}{|c|}{ Ano } \\
\hline & 1980 & 1981 & 1982 & 1983 & 1984 & 1985 & 1986 & 1987 & 1988 & 1989 \\
\hline I & $\mathrm{T} / \mathrm{S}$ & $\mathrm{T} / \mathrm{S}$ & $\mathrm{T} / \mathrm{S}$ & $\mathrm{T} / \mathrm{S}$ & $\mathrm{T} / \mathrm{S}$ & $\mathrm{T} / \mathrm{S}$ & $\mathrm{T} / \mathrm{S}$ & $\mathrm{T} / \mathrm{S}$ & $\mathrm{T} / \mathrm{S}$ & $\mathrm{T} / \mathrm{S}$ \\
\hline \multirow[t]{4}{*}{ II } & $\mathrm{T} / \mathrm{S}$ & $\mathrm{Co} / \mathrm{S}$ & $\mathrm{C} / \mathrm{S}$ & $\operatorname{Tr} / \mathrm{M}$ & $\mathrm{T} / \mathrm{S}$ & $\mathrm{Co} / \mathrm{S}$ & $\mathrm{C} / \mathrm{S}$ & $\mathrm{Se} / \mathrm{M}$ & $\mathrm{T} / \mathrm{S}$ & $\mathrm{Co} / \mathrm{S}$ \\
\hline & $\mathrm{Co} / \mathrm{S}$ & $\mathrm{C} / \mathrm{S}$ & $\mathrm{Tr} / \mathrm{M}$ & $\mathrm{T} / \mathrm{S}$ & $\mathrm{Co} / \mathrm{S}$ & $\mathrm{C} / \mathrm{S}$ & $\mathrm{Se} / \mathrm{M}$ & $\mathrm{T} / \mathrm{S}$ & $\mathrm{Co} / \mathrm{S}$ & $\mathrm{C} / \mathrm{S}$ \\
\hline & $\mathrm{T} / \mathrm{S}$ & $\mathrm{Tr} / \mathrm{M}$ & $\mathrm{T} / \mathrm{S}$ & $\mathrm{Co} / \mathrm{S}$ & $\mathrm{C} / \mathrm{S}$ & $\mathrm{Tr} / \mathrm{M}$ & $\mathrm{T} / \mathrm{S}$ & $\mathrm{Co} / \mathrm{S}$ & $\mathrm{C} / \mathrm{S}$ & $\mathrm{Se} / \mathrm{M}$ \\
\hline & $\mathrm{Tr} / \mathrm{S}$ & $\mathrm{T} / \mathrm{S}$ & $\mathrm{Co} / \mathrm{S}$ & $\mathrm{C} / \mathrm{S}$ & $\mathrm{Tr} / \mathrm{M}$ & $\mathrm{T} / \mathrm{S}$ & $\mathrm{Co} / \mathrm{S}$ & $\mathrm{C} / \mathrm{S}$ & $\mathrm{Se} / \mathrm{M}$ & $\mathrm{T} / \mathrm{S}$ \\
\hline \multirow[t]{3}{*}{ III } & $\mathrm{T} / \mathrm{S}$ & $\mathrm{Tv} / \mathrm{Tv}$ & $\mathrm{Tv} / \mathrm{M}$ & $\mathrm{T} / \mathrm{S}$ & $\mathrm{A} / \mathrm{S}$ & $\mathrm{E} / \mathrm{M}$ & $\mathrm{T} / \mathrm{S}$ & $\mathrm{A} / \mathrm{S}$ & $\mathrm{E} / \mathrm{M}$ & $\mathrm{T} / \mathrm{S}$ \\
\hline & $\mathrm{Tv} / \mathrm{Tv}$ & $\mathrm{Tv} / \mathrm{M}$ & $\mathrm{T} / \mathrm{S}$ & $\mathrm{Tv} / \mathrm{Tv}$ & $\mathrm{E} / \mathrm{M}$ & $\mathrm{T} / \mathrm{S}$ & $\mathrm{A} / \mathrm{S}$ & $\mathrm{E} / \mathrm{M}$ & $\mathrm{T} / \mathrm{S}$ & $\mathrm{A} / \mathrm{S}$ \\
\hline & $\mathrm{Tv} / \mathrm{M}$ & $\mathrm{T} / \mathrm{S}$ & $\mathrm{Tv} / \mathrm{Tv}$ & $\mathrm{Tv} / \mathrm{M}$ & $\mathrm{T} / \mathrm{S}$ & $\mathrm{A} / \mathrm{S}$ & $\mathrm{E} / \mathrm{M}$ & $\mathrm{T} / \mathrm{S}$ & $\mathrm{A} / \mathrm{S}$ & $\mathrm{E} / \mathrm{M}$ \\
\hline \multirow[t]{4}{*}{ IV } & $\mathrm{T} / \mathrm{S}$ & $\mathrm{Co} / \mathrm{S}$ & $\mathrm{L} / \mathrm{S}$ & $\mathrm{Tr} / \mathrm{M}$ & $\mathrm{T} / \mathrm{S}$ & $\mathrm{Co} / \mathrm{S}$ & $\mathrm{L} / \mathrm{S}$ & $\mathrm{Se} / \mathrm{M}$ & $\mathrm{T} / \mathrm{S}$ & $\mathrm{Co} / \mathrm{S}$ \\
\hline & $\mathrm{Co} / \mathrm{S}$ & $\mathrm{L} / \mathrm{S}$ & $\mathrm{Tr} / \mathrm{M}$ & $\mathrm{T} / \mathrm{S}$ & $\mathrm{Co} / \mathrm{S}$ & $\mathrm{L} / \mathrm{S}$ & $\mathrm{Se} / \mathrm{M}$ & $\mathrm{T} / \mathrm{S}$ & $\mathrm{Co} / \mathrm{S}$ & $\mathrm{L} / \mathrm{S}$ \\
\hline & $\mathrm{S}$ & $\mathrm{Tr} / \mathrm{M}$ & $\mathrm{T} / \mathrm{S}$ & $\mathrm{Co} / \mathrm{S}$ & $\mathrm{L} / \mathrm{S}$ & $\mathrm{Tr} / \mathrm{M}$ & $\mathrm{T} / \mathrm{S}$ & $\mathrm{Co} / \mathrm{S}$ & $\mathrm{L} / \mathrm{S}$ & $\mathrm{Se} / \mathrm{M}$ \\
\hline & M & $\mathrm{T} / \mathrm{S}$ & $\mathrm{Co} / \mathrm{S}$ & $\mathrm{L} / \mathrm{S}$ & $\mathrm{Tr} / \mathrm{M}$ & $\mathrm{T} / \mathrm{S}$ & $\mathrm{Co} / \mathrm{S}$ & $\mathrm{L} / \mathrm{S}$ & $\mathrm{Se} / \mathrm{M}$ & $\mathrm{T} / \mathrm{S}$ \\
\hline
\end{tabular}

${ }_{1}$ A: aveia branca; C: cevada; Co: colza; E: ervilhaca; L: linho; M: milho; S: soja; Se: serradela; Tr: tremoço; T: trigo; Tv: trevo vesiculoso.

Fonte: Santos et al. (1990) 
Pela análise da média variância da receita líquida dos dez anos (Tabela 3), os sistemas II (US\$ 302,22) e IV (US\$293,95) foram superiores significativamente aos sistemas I (US\$ 180,73) e III (US\$ 186,56). A análise da variância média da receita líquida permitiu separar os sistemas II e IV como as melhores alternativas a serem oferecidas aos agricultores, apresentando maior lucratividade. Resultados semelhantes foram obtidos por Santos et al. (1995b), ao pesquisar sistemas de rotação de culturas para trigo, sob sistema plantio direto, na região de Guarapuava, PR, durante dez anos.

Os dados da Tabela 4 foram gerados a partir da distribuição de probabilidade acumulada da distribuição normal dentro de cada sistema. O programa Biorisco divide essa distribuição em 20 intervalos de $5 \%$ de probabilidade cada. Esse tipo de análise pos- sibilita a escolha da alternativa com base numa determinada probabilidade de garantir uma renda líquida em um dado nível de escolha do tomador de decisão. Esse princípio basea-se no critério da "segurança em primeiro lugar", ou seja, qual a possibilidade de um dos sistemas apresentar uma renda líquida $\mathrm{x}$ ? $\mathrm{O}$ valor seria escolhido pelo tomador de decisão.

A análise da distribuição de probabilidade acumulada da receita líquida do sistema I, na baixa probabilidade de risco (5\%), revelou maior renda líqui$\mathrm{da} /$ ha (US\$2,33) do que os sistemas II (US\$ 0,32), e III e IV (US\$ -2,00). Na alta probabilidade de risco $(100 \%)$, o sistema III obteve a maior renda líquida/ha (US\$ 897,18), em comparação aos sistemas I (US\$ 499,18), II (US\$ 847,00) e IV (US\$ 861,01). Santos et al. (1995a), trabalhando com sistemas de rotação de culturas para cevada, sob sistema plantio di-

TABELA 2. Rendimento de grãos (kg/ha) de espécies que compõem os quatro sistemas de rotação de culturas para trigo. Embrapa-CNPT, Passo Fundo, RS, 1980 a $1989^{1}$.

\begin{tabular}{|c|c|c|c|c|c|c|c|c|c|c|}
\hline \multirow{2}{*}{$\frac{\text { Sistema }}{\mathrm{I}}$} & \multicolumn{2}{|c|}{1980} & \multicolumn{2}{|c|}{1981} & \multicolumn{2}{|c|}{1982} & \multicolumn{2}{|c|}{1983} & \multicolumn{2}{|c|}{1984} \\
\hline & $\mathrm{T}$ & S & $\mathrm{T}$ & S & $\mathrm{T}$ & S & $\mathrm{T}$ & S & $\mathrm{T}$ & S \\
\hline & 2.413 & 2.107 & 2.236 & 1.820 & 377 & 4.448 & 159 & 3.304 & 1.734 & 3.550 \\
\hline \multirow{8}{*}{ II } & $\mathrm{T}$ & $\mathrm{S}$ & Co & $\mathrm{S}$ & $\mathrm{C}$ & $\mathrm{S}$ & $\mathrm{Tr}$ & $\mathrm{M}$ & $\mathrm{T}$ & $\mathrm{S}$ \\
\hline & 2.541 & 1.986 & 1.628 & 1.307 & 1.157 & 4.753 & $*$ & 5.205 & 1.962 & 3.725 \\
\hline & Co & $\mathrm{S}$ & $\mathrm{C}$ & $\mathrm{S}$ & $\operatorname{Tr}$ & $\mathrm{M}$ & $\mathrm{T}$ & $\mathrm{S}$ & Co & $\mathrm{S}$ \\
\hline & 610 & 2.010 & 2.959 & 1.914 & $*$ & 4.260 & 1.493 & 3.489 & 1.244 & 3.491 \\
\hline & $\mathrm{T}$ & S & $\operatorname{Tr}$ & $\mathrm{M}$ & $\mathrm{T}$ & S & Co & S & $\mathrm{C}$ & $\mathrm{S}$ \\
\hline & 2.451 & 2.059 & $*$ & 2.373 & 1.045 & 4.410 & 1.009 & 3.469 & 2.192 & 3.734 \\
\hline & -2 & $\mathrm{~S}$ & $\mathrm{~T}$ & $\mathrm{~S}$ & Co & S & C & $\mathrm{S}$ & $\mathrm{Tr}$ & $\mathrm{M}$ \\
\hline & 2.494 & 2.975 & 1.747 & 874 & 3.027 & 2.258 & 3.279 & * & 5.854 & \\
\hline \multirow[t]{6}{*}{ III } & $\mathrm{T}$ & S & $\mathrm{Tv}$ & $\mathrm{TV}$ & $\mathrm{TV}$ & M & $\mathrm{T}$ & $\mathrm{S}$ & A & S \\
\hline & 2.509 & 1.998 & $*$ & $*$ & $*$ & 4.435 & 388 & 3.092 & $*$ & 3.523 \\
\hline & Tv & TV & Tv & M & $\mathrm{T}$ & $\mathrm{S}$ & $\mathrm{Tv}$ & Tv & E & $\mathrm{M}$ \\
\hline & * & $*$ & $*$ & 661 & 2.044 & 2.826 & * & $*$ & $*$ & 5.606 \\
\hline & $\mathrm{Tv}$ & $\mathrm{M}$ & $\mathrm{T}$ & $\mathrm{S}$ & $\mathrm{Tv}$ & $\mathrm{TV}$ & $\mathrm{Tv}$ & $\mathrm{M}$ & $\mathrm{T}$ & $S$ \\
\hline & $*$ & 5.897 & 2.686 & 1.764 & $*$ & $*$ & $*$ & 4.241 & 1.941 & 3.609 \\
\hline \multirow[t]{8}{*}{ IV } & $\mathrm{T}$ & S & Co & S & $\mathrm{L}$ & S & $\mathrm{Tr}$ & $\mathrm{M}$ & $\mathrm{T}$ & S \\
\hline & 2.315 & 2.001 & 1.554 & 1.420 & 576 & 4.303 & $*$ & 4.651 & 2.044 & 3.682 \\
\hline & $\mathrm{Co}$ & $\mathrm{S}$ & $\mathrm{L}$ & $\mathrm{S}$ & $\operatorname{Tr}$ & $\mathrm{M}$ & $\mathrm{T}$ & $\mathrm{S}$ & $\mathrm{Co}$ & $\mathrm{S}$ \\
\hline & 645 & 2.151 & 1.340 & 1.309 & $*$ & 3.937 & 1.784 & 3.421 & 1.164 & 3.415 \\
\hline & - & S & $\operatorname{Tr}$ & M & $\mathrm{T}$ & S & $\mathrm{Co}$ & $\mathrm{S}$ & L & S \\
\hline & 2.512 & $*$ & 2.029 & 2.117 & 2.444 & 1.065 & 3.373 & 1.354 & 3.781 & \\
\hline & - & $\mathrm{M}$ & $\mathrm{T}$ & $\mathrm{S}$ & $\mathrm{Co}$ & $\mathrm{S}$ & $\mathrm{L}$ & $\mathrm{S}$ & $\mathrm{Tr}$ & $\mathrm{M}$ \\
\hline & 7.183 & 2.766 & 1.790 & 776 & 2.831 & 1.166 & 3.474 & $*$ & 5.743 & \\
\hline
\end{tabular}


TABELA 2. Continuação

\begin{tabular}{|c|c|c|c|c|c|c|c|c|c|c|}
\hline \multirow{2}{*}{$\begin{array}{l}\text { Sistema } \\
\mathrm{I}\end{array}$} & \multicolumn{2}{|c|}{1985} & \multicolumn{2}{|c|}{1986} & \multicolumn{2}{|c|}{1987} & \multicolumn{2}{|c|}{1988} & \multicolumn{2}{|c|}{1989} \\
\hline & $\mathrm{T}$ & $S$ & $\mathrm{~T}$ & $S$ & $\mathrm{~T}$ & $\mathrm{~S}$ & $\mathrm{~T}$ & $\mathrm{~S}$ & $\mathrm{~T}$ & $S$ \\
\hline & 1.950 & 2.669 & 2.171 & 1.454 & 2.117 & 1.196 & 1.903 & 1.668 & 1.826 & 1.570 \\
\hline \multirow[t]{8}{*}{ II } & Co & $\mathrm{S}$ & $\mathrm{C}$ & $\mathrm{S}$ & $\mathrm{Se}$ & M & $\mathrm{T}$ & $\mathrm{S}$ & $\mathrm{Co}$ & $\mathrm{S}$ \\
\hline & 766 & 903 & 3.320 & 1.580 & * & 4.937 & 2.380 & 2.899 & 1.175 & 2.062 \\
\hline & $\mathrm{C}$ & $\mathrm{S}$ & $\mathrm{Se}$ & M & $\mathrm{T}$ & $\mathrm{S}$ & Co & $\mathrm{S}$ & $\mathrm{C}$ & $\mathrm{S}$ \\
\hline & 2.642 & 2.558 & $*$ & 5.082 & 2.508 & 1.770 & 1.477 & 2.023 & 3.433 & 2.009 \\
\hline & $\operatorname{Tr}$ & M & $\mathrm{T}$ & S & $\mathrm{Co}$ & $\mathrm{S}$ & $\mathrm{C}$ & S & $\mathrm{Se}$ & M \\
\hline & $*$ & 3.752 & 2.593 & 1.493 & 642 & 1.009 & 1.979 & 2.028 & $*$ & 7.132 \\
\hline & $\mathrm{T}$ & $\mathrm{S}$ & $\mathrm{Co}$ & $\mathrm{S}$ & $\mathrm{C}$ & $\mathrm{S}$ & $\mathrm{Se}$ & M & $\mathrm{T}$ & $\mathrm{S}$ \\
\hline & 2.547 & 2.725 & 1.407 & 1.306 & 2.799 & 1.373 & $*$ & 7.742 & 3.102 & 2.372 \\
\hline \multirow[t]{6}{*}{ III } & E & M & $\mathrm{T}$ & S & A & S & $\mathrm{E}$ & $\mathrm{M}$ & $\mathrm{T}$ & S \\
\hline & $*$ & 3.378 & 2.813 & 1.756 & $*$ & 1.449 & $*$ & 7.821 & 3.015 & 2.385 \\
\hline & $\mathrm{T}$ & S & A & S & E & M & $\mathrm{T}$ & S & A & S \\
\hline & 2.741 & 2.807 & $*$ & 1.768 & $*$ & 4.721 & 2.269 & 2.887 & * & 1.829 \\
\hline & A & S & E & M & $\mathrm{T}$ & S & A & $\mathrm{S}$ & E & $\mathrm{M}$ \\
\hline & $*$ & 2.585 & $*$ & 5.562 & 2.225 & 1.958 & $*$ & 2.250 & * & 6.984 \\
\hline \multirow[t]{8}{*}{ IV } & Co & $\mathrm{S}$ & $\mathrm{L}$ & $\mathrm{S}$ & $\mathrm{Se}$ & M & $\mathrm{T}$ & $\mathrm{S}$ & $\mathrm{Co}$ & $\mathrm{S}$ \\
\hline & 703 & 1.106 & 681 & 1.551 & $*$ & 5.011 & 2.483 & 3.124 & 1.064 & 2.091 \\
\hline & $\mathrm{L}$ & $\mathrm{S}$ & $\mathrm{Se}$ & $\mathrm{M}$ & $\mathrm{T}$ & $\mathrm{S}$ & $\mathrm{Co}$ & $\mathrm{S}$ & $\mathrm{L}$ & $\mathrm{S}$ \\
\hline & 836 & 2.772 & $*$ & 5.360 & 2.635 & 1.777 & 1.444 & 1.944 & 1.743 & 2.363 \\
\hline & $\operatorname{Tr}$ & $\mathrm{M}$ & $\mathrm{T}$ & $\mathrm{S}$ & $\mathrm{Co}$ & $\mathrm{S}$ & $\mathrm{L}$ & $\mathrm{S}$ & $\mathrm{Se}$ & $\mathrm{M}$ \\
\hline & $*$ & 3.776 & 2.768 & 1.752 & 723 & 1.109 & 1.850 & 1.978 & $*$ & 7.271 \\
\hline & $\mathrm{T}$ & S & $\mathrm{Co}$ & S & $\mathrm{L}$ & $\mathrm{S}$ & $\mathrm{Se}$ & M & $\mathrm{T}$ & $\mathrm{S}$ \\
\hline & 2.806 & 2.913 & 1.395 & 1.434 & 1.747 & 1.281 & $*$ & 7.898 & 2.998 & 2.572 \\
\hline
\end{tabular}

${ }^{1}$ A: aveia branca; C: cevada; Co: colza; E: ervilhaca; L: linho; M: milho; S: soja; Se: serradela; Tr: tremoço; T: trigo; Tv: trevo vesiculoso.

2 Não foi semeado nesse inverno.

* Cultura para cobertura de solo, no inverno, e para adubação verde, no verão.

Fonte: Santos et al. (1990)

TABELA3. Receita líquida média por hectare e por ano, em sistemas de rotação de culturas para trigo. Passo Fundo, RS, 1980 a 1989 .

\begin{tabular}{lcc}
\hline Sistema $^{2}$ & $\begin{array}{c}\text { Receita líquida média } \\
1980 \text { a } 1989\end{array}$ & Desvio-padrão \\
\hline & $-180,73 \mathrm{~b}$ & 112,93 \\
I & $302,22 \mathrm{a}$ & 192,98 \\
II & $186,56 \mathrm{~b}$ & 250,94 \\
III & $293,95 \mathrm{a}$ & 200,14 \\
IV &
\end{tabular}

${ }^{1}$ Médias seguidas da mesma letra, na vertical, não apresentam diferenças significativas, a 5\% de probabilidade, pelo teste de Duncan

2 I: trigo/soja; II: trigo/soja, colza/soja, cevada/soja e tremoço ou serradela/milho; III: trigo/soja, trevo/trevo e trevo/milho (1980-1983) e trigo/soja, aveia branca/soja e ervilhaca/milho (1984-1989); IV: trigo/soja, colza/soja, linho/soja e tremoço ou serradela/milho. reto, na região de Guarapuava, PR, obtiveram dados similares para a distribuição de probabilidade acumulada da receita líquida. Supondo-se que um agricultor "A" não queira correr risco superior a $5 \%$ de ter receita líquida negativa, ele jamais deverá escolher os sistemas III e IV. Por outro lado, um agricultor "B" que pretenda obter a maior renda líquida possível, escolheria o sistema III. Um agricultor " $C$ " que jogasse $50 \%$ de suas possibilidades de atingir a máxima receita líquida escolheria o sistema II para obter uma receita líquida menor ou igual a $\mathrm{R} \$ 293,91$ por hectare. Por esse método, a escolha da alternativa depende única e exclusivamente do nível de risco escolhido pelo tomador de decisão.

Mediante a análise da dominância estocástica, o sistema II domina os demais sistemas estudados (Tabela 5). Pelo método da dominância estocástica, o sistema II manteve-se como a melhor alternativa. Por sua vez, o sistema III dominou o sistema I, e o siste- 
ma IV dominou os sistemas I e III. Dados semelhantes foram obtidos por Santos et al. (1995b), pesquisando sistemas de rotação de culturas para trigo, sob sistema plantio direto, na região de Guarapuava, PR, durante dez anos. Trabalhando com sistemas denominados mistos, que integram lavoura e pecuária, sob sistema plantio direto, na região de Passo Fundo, RS, no período de três anos, Ambrosi \& Fontaneli (1994) separaram o sistema III [trigo/soja e pastagem consorciada (aveia preta + ervilhaca)/milho + soja] como o mais rentável e de menor risco, em relação aos demais sistemas estudados.

Observa-se que o sistema II mostrou-se, no âmbito do experimento, como a alternativa de menor risco, caso adotada pelos agricultores. Deve ser levado em conta que o risco tende a atuar como fator de impedimento à adoção de novas práticas agrícolas (Moutinho et al., 1978). Nesse caso, os sistemas alternativos (II e IV) foram escolhidos como os de menor risco, em comparação ao sistema I (monocultura trigo/soja), para a região de Passo Fundo.

O método da dominância estocástica apresentou nível maior de discriminação que o método da média variância e deve ser utilizado, sempre que possível, para testar novas recomendações aos agricultores. A utilização da dominância estocástica é mais um recurso matemático na escolha do sistema de produção. Neste estudo, destacou-se o sistema II ( $25 \%$ de trigo $/ 25 \%$ de soja, $25 \%$ de colza $/ 25 \%$ de soja, $25 \%$ de cevada $/ 25 \%$ de soja e $25 \%$ de leguminosas $/ 25 \%$ de milho) como o mais lucrativo e de menor risco.

Deve ser levado em consideração que o linho e a colza manifestaram baixos rendimentos de grãos em 1982, em 1985, em 1986 e em 1987, pelo excesso de precipitação pluvial no final da maturação e pela precipitação de granizo ocorrida próximo à colheita, respectivamente (Tabela 2) (Santos et al., 1987). Em 1982, o trigo em monocultura apresentou rendimento de grãos relativamente baixo em virtude da falta de rotação de culturas (Reis et al., 1983). Em 1983, houve redução no rendimento de grãos de trigo causado pela ocorrência de mosaico (Reis et al., 1985). Esses fatos repercutiram no resultado econômico, uma vez que as despesas foram maiores do que a receita líquida.
TABELA4. Distribuição de probabilidade acumulada da receita líquida (twentiles), por hectare, em sistemas de rotação de culturas para trigo. Passo Fundo, RS, 1980 a 1989.

\begin{tabular}{|c|c|c|c|c|}
\hline \multirow{2}{*}{$\begin{array}{l}\text { Probabilidade } \\
\text { acumulada (\%) }\end{array}$} & \multicolumn{4}{|c|}{ Sistema de rotação ${ }^{1}$} \\
\hline & I & II & III & IV \\
\hline & \multicolumn{4}{|c|}{ IS\$ } \\
\hline 0 & $-2,00$ & $-2,00$ & $-2,00$ & $-2,00$ \\
\hline 5 & 2,33 & 0,32 & $-2,00$ & $-2,00$ \\
\hline 10 & 26,09 & 38,56 & $-2,00$ & 22,58 \\
\hline 15 & 42,11 & 65,93 & $-2,00$ & 50,97 \\
\hline 20 & 86,03 & 140,98 & $-2,00$ & 128,80 \\
\hline 25 & 104,38 & 172,35 & 19,91 & 161,33 \\
\hline 30 & 116,04 & 192,28 & 45,82 & 182,00 \\
\hline 35 & 133,12 & 221,46 & 83,77 & 212,26 \\
\hline 40 & 145,51 & 242,63 & 111,30 & 234,22 \\
\hline 45 & 161,74 & 270,36 & 147,36 & 262,98 \\
\hline 50 & 175,52 & 293,91 & 177,98 & 287,40 \\
\hline 55 & 196,44 & 329,65 & 224,46 & 324,47 \\
\hline 60 & 221,41 & 372,34 & 279,96 & 368,74 \\
\hline 65 & 229,50 & 386,16 & 397,94 & 383,07 \\
\hline 70 & 240,92 & 405,68 & 323,31 & 403,32 \\
\hline 75 & 257,88 & 424,66 & 361,00 & 433,37 \\
\hline 80 & 280,18 & 472,76 & 410,55 & 472,89 \\
\hline 85 & 298,12 & 503,42 & 450,42 & 504,69 \\
\hline 90 & 323,66 & 547,06 & 507,16 & 549,95 \\
\hline 95 & 363,83 & 615,71 & 596,43 & 621,14 \\
\hline 100 & 499,18 & 847,00 & 897,18 & 861,01 \\
\hline
\end{tabular}

${ }^{1}$ I: trigo/soja; II: trigo/soja, colza/soja, cevada/soja e tremoço ou serradela/milho; III: trigo/soja, trevo/trevo e trevo/milho (1980-1983) e trigo/soja, aveia branca/soja e ervilhaca/milho (1984-1989); IV: trigo/soja, colza/soja, linho/soja e tremoço ou serradela/milho.

TABELA 5. Dominância estocástica dos sistemas de rotação de culturas para trigo. Passo Fundo, RS, 1980 a 19891.

\begin{tabular}{ccccc}
\hline \multirow{2}{*}{$\begin{array}{c}\text { Sistema } \\
\text { de rotação }^{2}\end{array}$} & I & II & III & IV \\
\cline { 2 - 5 } I & - & 0 & 0 & 0 \\
II & 1 & - & 1 & 1 \\
III & 1 & 0 & - & 0 \\
IV & 1 & 0 & 1 & - \\
\hline
\end{tabular}

1 A leitura deve ser feita no sentido horizontal: 0 (zero) significa que a tecnologia da linha é dominada pela da coluna, e 1 (um) que a tecnologia da linha domina a da coluna.

2 I: trigo/soja; II: trigo/soja, colza/soja, cevada/soja e tremoço ou serradela/milho; III: trigo/soja, trevo/trevo e trevo/milho (1980-1983) e trigo/soja, aveia branca/soja e ervilhaca/milho (1984-1989); IV: trigo/soja, colza/soja, linho/soja e tremoço ou serradela/milho. 
O sistema III, que contemplou trevo vesiculoso, de 1980 a 1983, e aveia branca, de 1984 a 1989 (Tabela 1), ficou inferiorizado em relação aos sistemas II e IV, pelo fato de as espécies terem servido apenas de cobertura de solo. Por essa razão, em três anos o sistema apresentou receita líquida negativa (Santos et al., 1995c).

\section{CONCLUSÃO}

Em termos de rentabilidade e risco, os sistemas podem ser classificados na seguinte ordem decrescente: sistema II, sistema IV, sistema III e sistema I.

\section{REFERÊNCIAS}

AMBROSI, I.; FONTANELI, R.S. Análise de risco de quatro sistemas alternativos de produção de integração lavoura/pecuária. Teoria e Evidência Econômica, Passo Fundo, v.2, n.1, p.129-148, 1994.

AMBROSI, I.; ZENTNER, R.P. Aspectos econômicos no sistema de manejo conservacionista. In: FERNANDES, J.M.; FERNANDEZ, M.R.; KOCHHANN, R.A.; SELLES, F.; ZENTNER, R.P. (Eds.). Manual de manejo conservacionista do solo para os estados do Rio Grande do Sul, Santa Catarina e Paraná. Passo Fundo: Embrapa-CNPT, 1991. p.63-69. (Embrapa-CNPT. Documentos, 1).

ANDERSON, J.R. Modeling decision-making under risk. México: CIMMYT, 1976.

BRASIL. Ministério da Agricultura. Departamento Nacional de Pesquisa Agropecuária. Divisão de Pesquisa Pedológica. Levantamento de reconhecimento dos solos do Rio Grande do Sul. Recife, 1973. 341p. (Boletim técnico, 30).

CRUZ, E.M. da. Aspectos teóricos sobre incorporação de riscos em modelos de decisão. In: CONTINI, E.; ARAÚJO, J.D. de; OLIVEIRA, A.J. de; GARRIDO, W.E. (Eds.). Planejamento da propriedade agrícola - modelos de decisão. 2.ed. Brasília: Embrapa-DDT, 1986. p.237-260. (Embrapa-DDT. Documentos, 7).

CRUZ, E.R. da. PACTA - Programa de avaliação comparativa de tecnologias alternativas: guia do usuário. Brasília: Embrapa-DDM, 1980.7p.
DERPSCH, R.; CALEGARI, A. Plantas para adubação verde de inverno. Londrina: IAPAR, 1992. 80p. (IAPAR. Circular, 73).

DERPSCH, R.; ROTH, C.H.; SIDIRAS, N.; KOPKE, U. Importância da rotação de culturas. In: DERPSCH, R.; ROTH, C.H.; SIDIRAS, N.; KOPKE, U. Controle da erosão no Paraná, Brasil: sistemas de cobertura do solo, plantio direto e preparo convencional do solo. Eschborn: GTZ/IAPAR, 1991. p.147164.

FELDSTEIN, M.S. Mean-variance analysis in the theory of liquidity preference and portfolio selection. Review of Economic Studies, Oxford, v.36, n.1, p.5-14, 1969.

HANOCH, G.; LEVY, H. Efficient portfolio selection with quadratic and cubic utility. Journal of Business, Chicago, v.43, n.2, p.181-189, 1970.

IGNACZAK, J.C.; ARIAS, G.; IORCZESKI, E.J. Produção de grãos de cevada corrigida em função de classificação comercial. In: REUNIÃO NACIONAL DE PESQUISA DE TRIGO, 11., 1980, Porto Alegre. Solos, ecologia, fisiologia e práticas culturais. Passo Fundo: Embrapa-CNPT, 1980. v.3, p.98100.

MOUTINHO, D.A.; SANDERS JUNIOR, J.H.; WEBER, M.T. Tomada de decisão sob condições de risco em relação à nova tecnologia para a produção de feijão de corda. Revista de Economia Rural, Brasília, v.16, n.4, p.41-58, 1978.

REIS, E.M.; SANTOS, H.P. dos; LHAMBY, J.C.B. Rotação de culturas. I. Efeito sobre doenças radiculares do trigo nos anos 1981 e 1982. Fitopatologia Brasileira, Brasília, v.8, n.3, p.431-437, 1983.

REIS, E.M.; SANTOS, H.P. dos; PEREIRA, L.R. Rotação de culturas. IV. Efeito sobre mosaico e doenças radiculares do trigo em 1983. Fitopatologia Brasileira, Brasília, v.10, n.3, p.637-642, 1985.

REUNIÃO DE PESQUISA DE SOJA DA REGIÃO SUL, 22., 1995, Porto Alegre. Recomendações técnicas para a cultura da soja no Rio Grande do Sul e Santa Catarina: 1995/96. Porto Alegre: UFRGS, Departamento de Plantas de Lavoura, 1995. 80p.

SANTOS, H.P. dos; AMBROSI, I.; SANDINI, I. Análise de risco de sistemas de rotação de culturas envolvendo soja e cevada, num período de dez anos, sob plantio direto, em Guarapuava, PR. In: REUNIÃO DE

Pesq. agropec. bras., Brasília, v.34, n.4, p.519-526, abr. 1999 
PESQUISA DE SOJA DA REGIÃO SUL, 23. 1995, Porto Alegre. Soja: resultados de pesquisa 1994-1995. Passo Fundo: Embrapa-CNPT, 1995a. p.164-173. (Embrapa-CNPT. Documentos, 22).

SANTOS, H.P. dos; AMBROSI, I.; WOBETO, C. Análise de risco de sistemas de rotação de culturas envolvendo soja e trigo, num período de dez anos, sob plantio direto, em Guarapuava, PR. In: REUNIÃO DE PESQUISA DE SOJA DA REGIÃO SUL, 23. 1995, Porto Alegre. Soja: resultados de pesquisa 1994-1995. Passo Fundo: Embrapa-CNPT, 1995b. p.193-202. (Embrapa-CNPT. Documentos, 22).

SANTOS, H.P. dos; IGNACZAK, J.C.; LHAMBY, J.C.B.; AMBROSI, I. Análise econômica de quatro sistemas de rotação de culturas envolvendo soja e trigo, num período de dez anos, em Passo Fundo, RS. Pesquisa Agropecuária Brasileira, Brasília, v.30, n.9, p.1167-1175, set. 1995c

SANTOS, H.P. dos; PEREIRA, L.R. Rotação de culturas em Guarapuava. XIV. Efeitos de sistemas de sucessão de culturas de inverno sobre algumas características agronômicas de milho, em plantio direto. Pesquisa Agropecuária Brasileira, Brasília, v.29, n.11, p.1691-1699, nov. 1994.

SANTOS, H.P. dos; REIS, E.M. Efeitos de culturas de inverno sobre o rendimento de grãos e sobre a estatura de plantas da soja. Pesquisa Agropecuária Brasileira, Brasília, v.26, n.5, p.729-735, maio 1991.
SANTOS, H.P. dos; REIS, E.M.; PEREIRA, L.R. Rotação de culturas. XVII. Efeitos no rendimento de grãos e nas doenças do sistema radicular do trigo de 1980 a 1987. Pesquisa Agropecuária Brasileira, Brasília, v.25, n.11, p.1627-1635, nov. 1990.

SANTOS, H.P. dos; REIS, E.M.; PEREIRA, L.R.; VIEIRA, S.A. Efeito da rotação de culturas no rendimento de grãos e na ocorrência de doenças radiculares do trigo (Triticum aestivum) e de outras culturas de inverno e de verão, de 1979 a 1986. Passo Fundo: Embrapa-CNPT, 1987. 38p. (Embrapa-CNPT. Documentos, 7).

SILVA, R.I. da; DHEIN, R.A. Viabilização sócio-econômica da rotação de culturas e da adubação verde na COTRIJUI. In: REUNIÃO CENTRO-SUL DE ADUBAÇÃO VERDE E ROTAÇÃO DE CULTURAS, 4., 1993, Passo Fundo, RS. Anais... Passo Fundo: Embrapa-CNPT, 1994. p.15-27. (EmbrapaCNPT. Documentos, 14).

ZENTNER, R.P.; SELLES, F.; SANTOS, H.P. dos; AMBROSI, I. Effect of crop rotations on yields, soil characteristics, and economic returns in Southern Brazil. In: INTERNATIONAL WORKSHOP ON CONSERVATION TILLAGE SYSTEMS, 1990, Passo Fundo. Conservation tillage for subtropical areas, proceedings. Passo Fundo: CIDA/EmbrapaCNPT, 1990. p.96-116. 